\title{
FATORES ASSOCIADOS AO DESMAME AOS QUATRO MESES EM BEBÊS DE MÃES ADOLESCENTES
}

\section{FACTORS ASSOCIATED WITH WEANING AT FOUR MONTHS IN BABIES OF ADOLESCENT MOTHERS}

\section{FACTORES ASOCIADOS AL DESMAME A LOS CUATRO MESES EN BEBÉS DE MADRE ADOLESCENTES}

\author{
Edficher Margotti ${ }^{1}$, Willian Margotti ${ }^{2}$
}

\section{RESUMO}

Objetivo: Apresentar os índices de aleitamento materno exclusivo e verificar os fatores associados ao desmame aos quatro meses, em mães adolescentes. Método: Estudo transversal com amostra composta por adolescentes de 13 à 18 anos e seus bebês; os nascimentos foram em hospitais amigo da criança, na capital Belém do Pará. $\mathrm{O}$ trabalho foi realizado com uma amostra de 92 adolescentes. A pré-seleção das adolescentes era feita na maternidade. A segunda etapa foi no quarto mês pós-parto, mantendo-se contato com as adolescentes, por telefone e verificado o tipo de alimentação que se encontrava o bebê. A análise descritiva foi apresentada mediante frequência simples, percentual e razão de chance. A relação entre o desfecho e o tipo de alimentação foi avaliada por regressão logística. O teste de Wald foi utilizado para testar a significância das variáveis. As diferenças foram significativas quando $\mathrm{p}<0,05$. As características das mães e das crianças foram comparadas pelo teste de Pearson e foram significativos se $\mathrm{p}<5 \%$. Resultados: $\mathrm{O}$ aleitamento materno misto aos quatro meses foi de $22,82 \%$, desmame de $17,40 \%$ e $59,78 \%$ foi de aleitamento materno exclusivo. Os fatores escolaridade, estado civil, trabalha fora, companheiro não incentiva o aleitamento estão significativamente relacionados negativamente ao aleitamento materno exclusivo. Conclusão: $\mathrm{O}$ índice de aleitamento materno exclusivo no quarto mês de vida dos bebês foi baixo, os fatores associados ao aleitamento materno exclusivo foram: baixa escolaridade, estado civil sem companheiro, mãe que trabalha fora de casa e o companheiro que não incentiva o aleitamento.

Descritores: Aleitamento Materno; Desmame; Medidas de Associação.

\footnotetext{
Enfermeira Obstetra. Doutora em Pediatria e Saúde da Criança, Docente do Semi Internato em Pediatria da Faculdade de Enfermagem da Universidade Federal do Pará (UFPA). Belém, PA, Brasil.

${ }^{2}$ Fisioterapeuta, Mestre em Saúde Coletiva, Docente da Faculdade de Fisioterapia da Universidade do Sul de Santa Catariana-UNISUL.
} 


\begin{abstract}
Objective: To present the exclusive breastfeeding indexes and to verify factors associated with weaning at four months in adolescent mothers. Method: A cross-sectional study with a sample composed of adolescents aged 13 to 18 years and their infants; births were in babyfriendly hospitals in the capital city of Belém, Pará. The study was carried out with a sample of 92 adolescents. The pre-selection of the adolescents was done in the maternity ward. The second stage was in the fourth month postpartum, staying in contact with the adolescents by telephone and verified the type of feeding the baby was receiving. The descriptive analysis was presented by simple frequency, percentage and odds ratio. The relationship between the outcome and the type of feed was assessed by logistic regression. The Wald test was used to test the significance of the variables. The differences were significant when $p<0.05$. The characteristics of the mothers and the children were compared by the Pearson test and were significant if $\mathrm{p}<5 \%$. Results: Mixed breastfeeding at four months was $22.82 \%$, weaning from $17.40 \%$ and $59.78 \%$ from exclusive breastfeeding. The factors schooling, marital status, work away from home, companion does not encourage breastfeeding are significantly related negatively to exclusive breastfeeding. Conclusion: Exclusive breastfeeding in the fourth month of life of infants was low; factors associated with exclusive breastfeeding were low schooling, marital status without a partner, mother working away from home, and companion who did not encourage breastfeeding.
\end{abstract}

Descriptors: Breast Feeding; Weaning; Measures of Association.

\title{
RESUMEN
}

Objetivo: Presentar los índices de lactancia materna exclusiva y verificar los factores asociados al destete a los cuatro meses, en madres adolescentes. Metodo: Estudio transversal con muestra compuesta por adolescentes de 13 a 18 años y sus bebés, los nacimientos fueron en hospitales amigo del niño, en la capital Belém do Pará. El trabajo fue realizado con una muestra de 92 adolescentes. La pre-selección de las adolescentes era hecha en la maternidad. La segunda etapa fue en el cuarto mes post parto, se mantuvo contacto con las adolescentes, por teléfono y verificado el tipo de alimentación que se encontraba el bebé. El análisis descriptivo fue presentado mediante frecuencia simple, porcentual y razón de probabilidad. La relación entre el desenlace y el tipo de alimentación fue evaluada por regresión logística. La prueba de Wald fue utilizada para probar la significancia de las variables. Las diferencias fueron significativas cuando $\mathrm{p}<0,05$. Las características de las madres y de los niños fueron comparadas por el test de Pearson y fueron significativos si $\mathrm{p}<5 \%$. Resultados: La lactancia materna mixta a los cuatro meses fue del $22,82 \%$, el destete del $17,40 \%$ y el $59,78 \%$ fue de lactancia materna exclusiva. Los factores de escolaridad, estado civil, trabajo fuera, compañero no incentiva la lactancia están significativamente relacionados negativamente con la lactancia materna exclusiva. Conclusión: El índice de lactancia materna exclusiva en el cuarto mes de vida de los bebés fue bajo, los factores asociados a la lactancia materna exclusiva fueron: baja escolaridad, estado civil sin compañero, madre que trabaja fuera de casa y el compañero que no incentiva la lactancia.

Descriptores: Lactancia materna; Destete; Medidas de Asociación. 


\section{INTRODUÇÃO}

O aleitamento materno (AM) é altamente nutritivo, supri todas as necessidades alimentares do infante durante os 4-6 primeiros meses de vida. Daí em diante permanece como valioso suplemento proteico à dieta infantil. Além desses elementos, o leite materno contém açúcar, gorduras, sais minerais e vitaminas essenciais ao bom crescimento e desenvolvimento da criança. ${ }^{1}$

No conjunto das capitais brasileiras, a duração mediana do aleitamento materno exclusivo (AME) aumentou um mês, passando de 23,4 dias para 54,1 dias. $\mathrm{Na}$ evolução da prática do AME em menores de 4 meses, segundo região e capital, a região norte apresentou prevalência de $57,4 \%$ e a capital Belém apresentou uma prevalência de $65,9 \%$, sendo a mais alta entre as demais capitais da região Norte. ${ }^{2}$

Algumas mudanças de características da população podem influenciar, favorecendo ou dificultando no avanço dos indicadores do AM. Dentre os fatores maternos que podem contribuir positivamente para menor proporção de aleitamento materno estão as mães adolescentes. Estudos mostram que há associação positiva entre menor idade e menor índice de AME. ${ }^{3}$ Um estudo transversal com 275 crianças menores de 6 meses de idade, residentes no município de Serrana, evidenciou que mães adolescentes apresentam mais chance para desmamar seus filhos precocemente. ${ }^{4}$

Dentre os estudos realizados sobre o aleitamento materno exclusivo em populações de adolescentes um estudo mostrou que apenas $48,8 \%$ amamentaram exclusivamente ao peito o bebê no momento da alta hospitalar ${ }^{5}$. Outro estudo apontou que $16 \%$ das mães adolescentes abandonaram o AME antes de completar um mês de vida dos bebês ${ }^{6}$. Dentre as mães adolescentes que amamentaram exclusivamente ao peito ao quarto mês dos bebês, um estudo apresentou $74,40 \%^{7} \mathrm{e}$ outro indicou $59,78 \%^{8}$, referentes à amamentação exclusiva ao sexto mês de vida dos bebês, um estudo expressou $13,8 \%{ }^{9}$ e outro evidenciou $62,9 \% .^{10}$

Os fatores de proteção para o aleitamento materno aos 60 dias de vida do bebê, foram: o tempo de estudo da mãe acima de oito anos, o nascer em hospital amigo da criança e mãe que não trabalha fora do lar e aos 120 dias foram apenas a idade da mãe acima de 20 anos. ${ }^{11} \mathrm{Um}$ estudo mostrou que no quarto mês as variáveis foram: menor escolaridade materna, ter voltado a trabalhar, não ter recebido orientações sobre amamentação no puerpério e não receber ajuda do companheiro com a criança foram preditivas para o desmame. ${ }^{12}$ Os fatores associados à prática de desmame antes dos 
seis meses de idade foram: trabalho materno fora de casa e primiparidade. Em menores de doze meses, os fatores associados ao aleitamento materno foram: mulheres com idade igual ou superior a 35 anos, escolaridade de terceiro grau, mais de um filho e em licença-maternidade. ${ }^{13}$ Estudos ainda mostram que mães adolescentes com filhos anteriores e com maior grau de instrução tem uma maior prevalência de AME. ${ }^{9}$

A capital Belém do Pará foi a capital com melhor índice de aleitamento materno da região norte, pesquisa realizada pelo Ministério da Saúde em 2009². Essa pesquisa não mencionou índices de aleitamento materno em mães adolescentes na capital, visto que há inúmeros fatores que estão envolvidos diretamente na falta de conhecimentos relativos à amamentação, conhecimentos esses que influenciam diretamente na execução desta prática entre as adolescentes.

Com base nas menções acima, e com a problemática gravidez na adolescência e desmame precoce, o presente estudo teve como objetivo apresentar os índices de AME e verificar os fatores associados ao desmame aos quatro meses, em bebês de mães adolescentes de 13 a 18 anos, nascidos em hospitais Amigo da Criança, da capital Belém.

\section{MATERIAL E MÉTODOS}

Este estudo faz parte de uma pesquisa mais ampla intitulada "O Desmame Precoce e a Escala Breastfeeding Self-Efficacy-Short Form aplicada nos hospitais Conveniados ao Sistema Único de Saúde-SUS da capital Belém e região metropolitana".

Estudo transversal analítico, composto por um grupo de mães adolescentes (13 à 18 anos de idade) e seus respectivos bebês, todos os nascimentos foram em hospitais credenciados como amigo da criança, localizados na capital Belém do Pará, Brasil, nos anos de 2016 e 2017.

O cálculo amostral teve como valor crítico associado ao grau de confiança da amostra de 95,0\%, um erro amostral de + ou - $5 \%$, tendo a fórmula para o cálculo do tamanho da amostra, para uma estimativa confiável da proporção populacional, com $p$ e $q$ desconhecidos: $n=Z_{x / 2}^{2} \times p . q / E^{2}$, obtendo uma amostra estimada de 90 adolescentes.

O trabalho foi realizado em uma população de 92 adolescentes e seus respectivos bebês.

Para o estabelecimento das participantes ao estudo, a seleção das puérperas foi feita de forma contínua, simultânea e aleatória, conforme aconteciam os partos nos centros 
obstétricos dos hospitais. A pré-seleção das adolescentes puérperas era feita na maternidade, por uma aluna bolsista devidamente treinada e capacitada pelo pesquisador responsável.

Os critérios de inclusão utilizados foram: crianças nascidas com peso $>2.500 \mathrm{~g}$; nascidas de parto normal ou cesariana sem intercorrências pediátricas e/ou obstétricas; com idade gestacional acima de 36 semanas, crianças nessas condições geralmente são colocadas ao seio materno nas primeiras horas de vida e vão para o alojamento conjunto; mães residentes em zonas urbanas da capital, esse critério facilitaria a visita domiciliar, caso fosse necessário e que estivessem amamentando exclusivamente ao seio materno até o momento da alta hospitalar, por ser esse o tema da pesquisa.

Os critérios de exclusão foram: gemelares, recém-nascidos de mães soropositivas para o Vírus da Imunodeficiência Humana, bebês que por algum motivo não poderiam ser amamentadas ao peito materno, com malformações congênitas que interferissem na amamentação, bebês para adoção, crianças nessas condições geralmente são alimentadas com fórmulas lácteas e/ou através de dispositivos como sondas gástricas, enterais ou cirúrgicas; mães adolescentes com alguma limitação cognitiva e mental, pois a comunicação seria dificultosa e as adolescentes cujos responsáveis não autorizassem e não assinassem o termo de consentimento livre e esclarecido.

A primeira etapa acontecia na maternidade, onde eram abordadas após os nascimentos. E no momento anterior à alta hospitalar, respondiam questões de cunho sócio demográficos como escolaridade, renda, estado civil, se trabalha fora de casa, se o companheiro incentiva o aleitamento materno e se mora com mãe ou sogra.

Do prontuário foram coletados dados como a idade gestacional, o número de consultas de pré-natal, sexo e peso do recém-nascido e se o bebê havia mamado na sala de parto ou dentro das seis primeiras horas de vida.

Os participantes assinaram o termo de consentimento livre e esclarecido antes de qualquer procedimento do estudo. As menores de idade assinaram termo de assentimento e o termo de consentimento referente à sua participação foi assinado pelos pais ou responsáveis.

A segunda etapa se deu aos quatro meses de vida do bebê, quando se manteve contato com as mães adolescentes, por telefone e verificado o tipo de alimentação que se encontrava o bebê.

Duas perguntas foram feitas ao telefone: $1^{\text {a }}$. Se ela ainda estava dando leite apenas e exclusivamente do peito; e $2^{\text {a }} \mathrm{Se}$ ela já tinha começado a oferecer sucos, 
águas, chás, quaisquer outras fórmulas lácteas e se já tinha dado ao bebê algum tipo de comida, como "papinhas", tanto salgadas ou doces, raspas de frutas, caldos ou polpas de frutas. A segunda pergunta eliminava dúvidas quanto à resposta da primeira pergunta. Caso a resposta para a segunda pergunta fosse sim, essa criança deixava de ser do grupo do aleitamento materno exclusivo, passando para o grupo de aleitamento materno.

Foram analisados os dados em três grupos de estudo: Aleitamento Materno Exclusivo para crianças que receberam apenas leite materno como fonte de hidratação e alimentação, nenhum outro líquido ou sólido, à exceção de suplementos vitamínicos ou medicamentos; Aleitamento Materno para crianças que receberam, além do leite materno, outras fontes de hidratação e alimentação, como chás, sucos, caldos, papas e outros tipos de leite e Desmame em que o aleitamento materno exclusivo fosse interrompido antes do quarto mês de vida.

As visitas domiciliares ocorriam caso houvesse dificuldades em contatar a mãe por telefone.

As variáveis estudadas foram: idade gestacional, tipo de parto, consultas de pré natal, primigestação, bebê mamou na sala de parto ou dentro das primeiras 6 horas, incentivo ao aleitamento materno pelo companheiro, sexo e peso do recémnascido, escolaridade materna, mãe que trabalha fora do lar, renda, estado civil, morar com mãe e/ou sogra.

Para análise descritiva, os dados foram apresentados mediante frequência simples, percentual e razão de chance, conforme a simetria das variáveis. A relação entre a variável de desfecho tipo de alimentação ao quarto mês de vida da criança e os potenciais fatores associados para o aleitamento materno exclusivo foram avaliados por meio de regressão logística, tanto nas análises bivariadas, quanto nas multivariadas. O teste de Wald foi utilizado para testar a significância de cada variável no modelo. Para o modelo multivariável, consideraram-se, inicialmente, todas as covariáveis que apresentaram valores de $\mathrm{p}<0,10$. O procedimento a seguir foi a exclusão, de maneira individual, das covariáveis que apresentassem valores críticos de $p$ (valores maiores e não significativos). Esse procedimento foi repetido até que todas as variáveis no modelo apresentassem valores de $p<0,05$. Todos os testes foram bidirecionais, e as diferenças foram consideradas significativas com valor de $\mathrm{p}<0,05$. As características das mães e das crianças foram comparadas com o teste do Qui quadrado de Pearson e adotaram-se como significativos os valores de $\mathrm{p}<5 \%$. 
A pesquisa foi conduzida de acordo com a Declaração de Helsinki revisada em 2008 e foi aprovada pelo Comitê de Ética em Pesquisa da Universidade Federal do Pará-UFPA, sob o parecer de n 1.259.717, no dia 06 de outubro de 2015. O estudo respeitou as exigências formais contidas nas normas nacionais (Resolução CNS 466, de 12 de dezembro de 2012 do Conselho Nacional de Saúde) e internacionais regulamentadoras de pesquisas envolvendo seres humanos.

\section{RESULTADOS}

Foram entrevistadas 92 adolescentes, entre 13 e 18 anos de idade, sendo a idade média de 16,30 anos. A faixa de escolaridade mais representada foi $1^{\circ} \mathrm{Grau}$
Incompleto, referido por $52,17 \%$ das adolescentes, a maioria, 54,35\%, recebiam até um salário mínimo, eram amasiadas $55,43 \%$, não trabalhavam fora, 91,30\%, referiram morar com mãe/sogra 64,13\% e disseram ter o apoio do companheiro no ato de amamentar 68,48\%. O teste do Qui quadrado de Pearson $(\mathrm{p}<0.05)$ mostra que as proporções diferem significativamente entre os grupos de cada variável. Sendo que todas as variáveis sócio demográficas das mães adolescentes diferiram entre si no seu grupo; escolaridade, renda mensal, estado civil e trabalha fora de casa com $\mathrm{p}<0.0001$, a variável mora com mãe ou sogra foi de 0.0067 e companheiro incentiva no aleitamento foi de 0.0004 (Tabela 1).

Tabela 1. Perfil Sócio demográfico das mães adolescentes. Belém, Pará, 2016- 2017

\begin{tabular}{|c|c|c|c|}
\hline Escolaridade & $\mathbf{N}$ & $\%$ & $p$-valor \\
\hline $1^{\circ}$ Grau completo & 9 & 9,78 & \multirow{6}{*}{$<0.0001 *$} \\
\hline $1^{\circ} \mathrm{Grau}$ incompleto & 48 & 52,17 & \\
\hline $2^{\circ}$ Grau completo & 18 & 19,57 & \\
\hline $2^{\circ}$ Grau incompleto & 15 & 16,30 & \\
\hline $3^{\circ} \mathrm{Grau}$ completo & 1 & 1,09 & \\
\hline $3^{\circ}$ Grau incompleto & 1 & 1,09 & \\
\hline \multicolumn{4}{|l|}{ Renda Mensal } \\
\hline Até $1 \mathrm{SM}$ & 50 & 54,35 & \multirow{4}{*}{$<0.0001 *$} \\
\hline $1 \mathrm{a} 2 \mathrm{SM}$ & 24 & 26,09 & \\
\hline 2 a $3 \mathrm{SM}$ & 15 & 16,30 & \\
\hline 3 a 5 SM & 3 & 3,26 & \\
\hline \multicolumn{4}{|l|}{ Estado Civil } \\
\hline Solteira & 38 & 41,30 & \multirow{3}{*}{$<0.0001 *$} \\
\hline Casada & 3 & 3,26 & \\
\hline Amasiada & 51 & 55,43 & \\
\hline \multicolumn{3}{|l|}{ Trabalha fora de casa } & \\
\hline Sim (autônoma, Empregada doméstica e faxineira) & 8 & 8,70 & \multirow{2}{*}{$<0.0001 *$} \\
\hline Não & 84 & 91,30 & \\
\hline \multicolumn{3}{|l|}{ Mora com mãe ou sogra } & \\
\hline Sim & 59 & 64,13 & \multirow{2}{*}{$0.0067 *$} \\
\hline Não & 33 & 35,87 & \\
\hline
\end{tabular}




\begin{tabular}{l|l|l|l}
\hline Companheiro incentiva no aleitamento & \multicolumn{3}{l}{} \\
\hline Sim & 63 & 68,48 & $0.0004^{*}$ \\
\hline Não & 29 & 31,52 & \\
\hline \hline
\end{tabular}

Teste Qui-quadrado de Pearson para Proporções $(\mathrm{p}<0.05)$

*As proporções diferem significativamente.

A maioria dos bebês nascidos foram do sexo feminino $55,43 \%$, de parto normal $71,74 \%, 45,65 \%$ tiveram bebês com idade gestacional a termo, com peso ideal cerca de $97,83 \%$, a maioria dos bebês tiveram um bom Apgar no 5', cerca de 93,48\% e mamaram na sala de parto ou dentro das seis primeiras horas $93,48 \%$. As adolescentes que tiveram seus primeiros filhos foram de $77,17 \%$. As adolescentes que já tinham filhos, a maioria tinha apenas um filho $66,67 \%$ e tinham amantado muito no peito os filhos anteriores $66,67 \%$. Cerca de $63,04 \%$ das adolescentes realizaram até seis consultas de pré-natal. O teste do Qui quadrado de Pearson $(\mathrm{p}<0.05)$ mostra que as proporções diferem significativamente entre os grupos de cada variável, sendo que a variável sexo do Recém-nascido não tem diferença significativa entre as proporções verificadas no sexo masculino e no sexo feminino. Porém, as variáveis obstétricas que apresentaram maior diferença significativa entre o seu grupo foram: Bebê mamou na sala de parto ou dentro das seis horas de vida, tipo de parto e $\mathrm{n}^{\circ}$ de consultas pré natal, que foram $<0.0001$, 0.0003 e 0.0009 , respectivamente (Tabela 2).

Tabela 2. Perfil Obstétrico das mães adolescentes. Belém, Pará, 2016- 2017

\begin{tabular}{|c|c|c|c|}
\hline Variáveis & $\mathbf{N}$ & $\%$ & $p$-valor \\
\hline \multicolumn{4}{|l|}{ Sexo do RN } \\
\hline $\begin{array}{l}\text { Feminino } \\
\text { Masculino }\end{array}$ & $\begin{array}{l}51 \\
41\end{array}$ & $\begin{array}{l}55,43 \\
44,57 \\
\end{array}$ & 0.9654 \\
\hline \multicolumn{4}{|l|}{ Tipo de Parto } \\
\hline $\begin{array}{l}\text { Normal } \\
\text { Cesárea }\end{array}$ & $\begin{array}{l}66 \\
26\end{array}$ & $\begin{array}{l}71,74 \\
28,26 \\
\end{array}$ & $0.0003 *$ \\
\hline \multicolumn{4}{|c|}{$\begin{array}{l}\text { Bebê mamou na sala de parto ou dentro das seis } \\
\text { horas de vida }\end{array}$} \\
\hline $\begin{array}{l}\text { Sim } \\
\text { Não }\end{array}$ & $\begin{array}{l}86 \\
6\end{array}$ & $\begin{array}{l}93,48 \\
6,52\end{array}$ & $<0.0001 *$ \\
\hline \multicolumn{4}{|l|}{$\mathbf{N}^{\circ}$ de Consultas Pré-Natal } \\
\hline Até 6 consultas & 58 & 63,04 & \multirow{3}{*}{$0.0009 *$} \\
\hline 7 ou mais consultas & 27 & 29,35 & \\
\hline Não Fez Pré natal & 7 & 7,61 & \\
\hline
\end{tabular}

Com relação ao aleitamento materno aos quatro meses de vida do bebê, $17,40 \%$ estavam em desmame, $22,82 \%$ estavam em aleitamento materno, e 59,78\% encontravam-se em AME. O teste do Qui quadrado de Pearson $(\mathrm{p}<0.05)$ mostra que 
as proporções diferem significativamente entre si no grupo da variável Aleitamento ao $4^{\circ}$ mês, apresentando diferenças significativas entre as proporções do
Aleitamento Materno exclusivo, o Aleitamento Materno e o Desmame (Tabela 3).

Tabela 3. Indicadores de Aleitamento Materno Exclusivo ao quarto mês, em mães adolescentes. Belém, Pará, Brasil, 2016- 2017

\begin{tabular}{l|l|l|l}
\hline \hline Aleitamento ao $\mathbf{4}^{\mathbf{0}}$ Mês & $\mathbf{N}$ & $\boldsymbol{\%}$ & $\boldsymbol{p}$-valor \\
\hline Aleitamento Materno Exclusivo & 55 & 59,78 & \\
\cline { 1 - 2 } Aleitamento Materno & 21 & 22,82 & \multirow{2}{*}{$<0.0001^{*}$} \\
\cline { 1 - 2 } Desmame & 16 & 17,40 & \\
\hline \hline
\end{tabular}

Teste Qui-quadrado de Pearson para Proporções $(\mathrm{p}<0.05)$

*As proporções diferem significativamente.

A tabela que contém as variáveis fornece os resultados do teste de Wald para todas as variáveis incluídas no modelo. Assim sendo, os fatores escolaridade, estado civil, trabalha fora de casa e companheiro não incentiva no aleitamento contribuem significativamente para a explicação do modelo, ou seja, estão significativamente relacionados e influenciam negativamente no aleitamento materno exclusivo.

Tabela 4. Associação entre o aleitamento materno exclusivo e seus potenciais fatores que influenciam negativamente, em mães adolescentes com bebês menores de quatro meses. Belém, Pará, 2016- 2017

\begin{tabular}{|c|c|c|c|}
\hline Variáveis & $\begin{array}{l}\text { Razão de } \\
\text { Chance }\end{array}$ & IC $95 \%$ & $p$-valor \\
\hline Escolaridade (Baixa) & 0,981 & $0,963-0,999$ & $0,031^{*}$ \\
\hline Renda (1 a 3 salários mínimos) & 0,961 & $0,765-1,206$ & 0,822 \\
\hline Estado Civil (sem companheiro) & 0,986 & $0,973-1,000$ & $0,040 *$ \\
\hline Trabalha fora de casa & 1,061 & $1,034-1,088$ & $0,000 *$ \\
\hline $\begin{array}{l}\text { Bebê mamou na sala de parto } \\
\text { ou dentro das seis horas de vida }\end{array}$ & 0,967 & $0,862-1,076$ & 0,544 \\
\hline Tipo de parto (cesária) & 1,001 & $0,999-1,003$ & 0,246 \\
\hline Não Fez Pré Natal & 0,987 & $0,912-1,007$ & 0,843 \\
\hline Mora com mãe ou sogra & 0,917 & $0,716-1,17$ & 0,455 \\
\hline Companheiro Não incentiva o aleitamento & 1,588 & $1,132-2,288$ & $0,010 *$ \\
\hline
\end{tabular}

\section{DISCUSSÃO}

No presente estudo, trabalhar fora de casa contribuiu negativamente para o
AME, o que condiz com achado de outros trabalhos, com mulheres de várias faixas etárias $^{14,15,16}$, nos quais a permanência da mulher em casa foi fator positivo e, 
portanto, facilitador ao sucesso da amamentação. Contudo, há controvérsias sobre a relação entre desmame precoce e trabalho fora de casa. Trabalhar fora de casa mostrou-se associada ao aleitamento materno aos 60 dias, mas sem associação significativa com o aleitamento aos 120 dias. $^{11}$

Nos resultados deste estudo, a escolaridade baixa evidenciou-se como um fator associado negativamente para a amamentação exclusiva. Indo de encontro a outro achado, onde apresentou que adolescentes com maior nível de escolaridade apresentam maior prevalência de AME. ${ }^{9}$ Provavelmente, mulheres sem instrução ou com pouca instrução desconhecem a importância do aleitamento materno exclusivo até no mínimo seis meses de vida, pois não sabem do alto valor nutritivo e calórico do leite materno ao recém-nascido, e nem dos nutrientes necessários ao seu crescimento e desenvolvimento. A maioria dos trabalhos realizados com mulheres de todas as idades demonstra que o aleitamento materno é efetivo nas mulheres de maior escolaridade..$^{3,13,14,15}$ Entretanto, outro relato $^{17}$ mostrou maior prevalência de interrupção do aleitamento materno exclusivo entre as mulheres de maior escolaridade.

O resultado chama atenção, uma vez que a falta de apoio do companheiro na amamentação tem relação estatística positiva no desmame do bebê e consequentemente prejudica na manutenção e duração do AM pela adolescente. Coincidindo com os resultados encontrados em outros trabalhos, ${ }^{14,16,18,19}$ onde demonstra que o reflexo deste aumento no apoio paterno ao aleitamento materno, proporcionalmente com o passar dos anos romperá o modelo tradicional de paternidade, uma vez que o mesmo exerce influência positiva para a decisão em amamentar e a duração da amamentação.

No presente estudo, a prevalência de aleitamento materno exclusivo, AME, entre bebês de quatro meses de vida, de mães adolescentes de 13 a 18 anos de idade foi de $59,78 \%$, sendo que $100,0 \%$ estavam em AME no momento da alta hospitalar. Nosso achado vai de encontro aos dados de outras pesquisas com adolescentes, como a realizada na cidade de Dois Córregos, SP, onde 74,40\% das adolescentes, de 14 a 19 anos de idade, amamentaram exclusivamente ao peito até o quarto mês de vida dos bebês ${ }^{7}$ e a realizada em Quito no Equador, onde as adolescentes apresentaram uma prevalência de $62,9 \%$ de $\mathrm{AME}$ ao sexto mês. ${ }^{10}$ Isso nos deixa claro que não é pelo fato de ser adolescente, menor de idade, que a mulher não pode ser responsável para com cuidado do seu filho e consciente 
a respeito do ato e da importância em amamentar ao peito os seus filhos menores de 4 meses de vida

Nosso resultado também vai de encontro aos estudos de abrangência nacional, com mães de todas as idades, onde a prevalência de aleitamento materno exclusivo entre os quatro meses foi de $49,8 \%{ }^{20}, 53,7 \%{ }^{13}$ e de $65,9 \% .^{2}$

Outros trabalhos com mães menores de vinte anos de idade apresentaram resultados distintos aos nossos, com relação ao AME aos quatro meses, com prevalência de $20,0 \%{ }^{2}, 31,4 \%$ de $\mathrm{AME}^{9}$ e de $40,0 \% .^{20} \mathrm{~A}$ incidência de abandono do AME no quarto mês, em nossos achados, foi de 17,4\%. Resultados em contrário também foram obtidos, filhos de adolescentes de 13 a 19 anos de idade, que tiveram uma incidência de desmame no quarto mês de $69,6 \%{ }^{12}$ Isso nos remete a analisar com urgência os perfis das adolescentes de cada região, pois os dados se mostram conflitantes com relação a amamentação na adolescência; não se sabe se isso tem relação com a cultura e os costumes de cada região.

O presente estudo traz contribuições significativas para a saúde e para a enfermagem, considerando que as atividades de promoção à saúde da adolescente e da criança influenciam diretamente nos índices de AME, método esse inquestionável para a saúde da criança. Este estudo apresenta resultados relevantes que devem ser avaliados pelo poder público, a fim de diminuir os índices de desmame precoce entre as mães adolescentes, não só da região norte, mas de todo território brasileiro.

\section{CONCLUSÃO}

$\mathrm{O}$ estudo concluiu que os fatores associados negativamente ao aleitamento materno exclusivo foram: baixa escolaridade, estado civil sem companheiro, mãe que trabalha fora de casa e o companheiro que não incentiva o aleitamento materno.

O estudo também revelou que o índice de aleitamento materno exclusivo no quarto mês de vida dos bebês foi considerado baixo, lembrando que nossa população de adolescentes teve como critério de inclusão a amamentação exclusiva no momento da alta hospitalar.

As conclusões da presente pesquisa podem contribuir para a reflexão em outras realidades semelhantes, visto que a metodologia comum a outros estudos foi utilizada aqui. Tais reflexões podem apontar caminhos para a superação das dificuldades enfrentadas pelos profissionais dos serviços de saúde no apoio à adolescente que amamenta e a sua família.

Evidencia-se algumas limitações para o desenvolvimento do estudo: a 
informação sobre o desfecho, ou seja, sobre modificações no esquema alimentar da criança aos quatro meses de vida; pode em algum momento ter sido distorcida pela mãe adolescente, e promovido à classificação em categorias de exposição alteradas. Esse fato pode ocorrer ainda que regras claras tenham sido definidas para a classificação da exposição e do desfecho. $\mathrm{O}$ viés da memória, que as adolescentes podem ter apresentado com relação ao período de desmame. O viés de falsa resposta ou de não aceitação da situação, onde as adolescentes podem não relatar que não estão conseguindo amamentar, já que pode trazer constrangimento pessoal e gerar embaraço.

\section{REFERÊNCIAS}

1. Rezende J. Obstetrícia fundamental. 12ed. Rio de Janeiro: Guanabara Koogan; 2012.

2. Ministério da Saúde (Brasil), Secretaria de Atenção à Saúde, Departamento de Ações Programáticas e Estratégicas. II Pesquisa de prevalência de aleitamento materno nas capitais brasileiras e Distrito Federal. Brasília, DF: Ministério da Saúde; 2009.

3.Vieira GO, Reis MR, Vieira TO, Oliveira NF, Silva LR, Giugliani ER. Trends in breastfeeding indicators in a city of northeastern Brazil. J. Pediatri (Rio J). 2015; 91(3):270-77.

4. Queluz MC, Pereira MJB, Santos CB, Leite AM, Ricco RG. Prevalência e determinantes do aleitamento materno exclusivo no município de Serrana, São
Paulo, Brasil. Rev Esc Enferm USP. 2012; 46(3):537-43.

5. Leclair E, Robert N, Sprague AE, Fleming N. Factors associated with breastfeeding initiation in adolescent pregnancies: a cohort study. J Pediatr Adolesc Gynecol. Dec 2015; 28(6):516-21. 6. Gómez LY, Díaz CE, Manrique R. Factores asociados con lactancia materna exclusiva hasta el sexto mes en madres adolescentes. Medellin, 2010. Rev Salud Pública. 2013; 15(3):374-85.

7. Filamingo BO, Lisboa BCF, Basso NAS. A prática do aleitamento materno entre mães adolescentes na cidade de Dois Córregos, estado de São Paulo. Sci Med. 2012; 22(2):81-85.

8. Margotti E, Margotti W. Mães adolescentes e aleitamento materno até quatro meses. Inova Saúde. 2017; 6(2):7387.

9. Gusmão AM, Béria JU, Gigante LP, Leal AF, Schermann LB. Prevalência de aleitamento materno exclusivo e fatores associados: estudo transversal com mães adolescentes de 14 a 16 anos em Porto Alegre, RS, Brasil. Ciênc Saúde Colet. 2013; 18(11):3357-68.

10. Jara-Palácios MA, Cornejo AC, Peláez GA, Verdesoto J, Galvis AA. Prevalence and determinants of exclusive breastfeeding among adolescent mothers from Quito, Ecuador: a cross-sectional study . Int Breastfeed J. 2015; 10:33.

11. Margotti E, Epifanio M. Aleitamento materno exclusivo e a Escala de Autoeficácia na Amamentação. Rev Rene. set/out 2014; 15(5):771-9.

12. Machado MCM, Assis KF, Oliveira FCC, Ribeiro AQ, Araújo RMA, Cury AF, et al. Determinantes do abandono do aleitamento materno exclusivo: fatores psicossociais. Rev Saúde Pública. 2014; 48(6):985-94.

13. Souza SNDH, Migoto MT, Rossetto EG, Mello DF. Prevalence of breastfeeding and associated factors in the municipality of Londrina (PR, Brazil). Acta Paul Enferm. 2012; 25(1):29-35. 
14. Margotti E, Margotti W. 2017. Fatores relacionados ao aleitamento materno exclusivo em bebês nascidos em hospital amigo da criança em uma capital do norte brasileiro. Saúde Debate. jul/set 2017; 41(114):860-71.

15. Asemahagn MA. Determinants of exclusive breastfeeding practices among mothers in azezo district, northwest Ethiopia. Int Breastfeed J. 2016; 11:22. 16. Tewabe T, Mandesh A, Gualu T, Alem G, Mekuria G, Zeleke H. Exclusive breastfeeding practice and associated factors among mothers in Motta town, East Gojjam zone, Amhara Regional State, Ethiopia, 2015: a cross-sectional study. Int Breastfeed J. 2017; 12:12.

17. Campos AMS, Chaoul CO, Carmona EV, Higa R, Vale IN. Prática de aleitamento materno exclusivo informado pela mãe e oferta de líquidos aos seus filhos. Rev Latinoam Enferm. 2015; 23(2): 283-90.

18. Silva BT, Santiago LB, Lamonier JA. Apoio paterno ao aleitamento materno: uma revisão integrativa. Rev Paul Pediatr. 2012; 30(1):122-30.

19. Salvador JP, Ximenes VL, Silva ICM, Silva MFP. Participação do companheiro na promoção do aleitamento materno exclusivo em hospital amigo da criança. Rev Interdisciplin NOVAFAPI. jan/fev/mar 2012; 5(1):30-36. 20. Sadeck LSR, Leoni CR. Avaliação da situação do aleitamento materno em menores de um ano de idade no Município de São Paulo, Brasil, em 2008. Cad Saúde Pública. 2013; 29(2):397-402.

RECEBIDO: 23/08/2018

APROVADO: $14 / 12 / 2018$ PUBLICADO: $12 / 2018$ 